\title{
PSYCHE
}

\begin{tabular}{lll}
\hline Vol. 86 & December, 1979 & No. 4 \\
\hline
\end{tabular}

\section{WORKER COMPATIBILITIES WITHIN AND BETWEEN POPULATIONS OF RHYTIDOPONERA METALLICA*}

\author{
By Caryl P. Haskins, Edna F. Haskins \\ Haskins Laboratories, Inc., \\ New Haven, Connecticut 06510
}

\begin{abstract}
INTRODUCTION
The markedly diffuse nature of the colonial structure of Rhytidoponera metallica, associated with the rarity of true females and the usual presence of multiple fertilized egg-laying "workers" in the community, blurs the spatial distinction between naturally occurring "colonies" to an unusual degree. This condition coupled with the indefinite nest-form frequently typical of the species, which commonly occupies leaf-litter and superficial layers of soil, makes it difficult on occasion to distinguish a mosaic of neighboring "colonies" from what often superficially appears as merely a rather highly "viscous" population of socialized individuals. This situation lends special interest to the question of how far one mechanism commonly involved in the maintenance of integrity in formicid communities, that of colony "identification" among workers, is developed in $R$. metallica, and, if it is developed, what may be its basic characteristics. This interest has led to a preliminary investigation of those questions over the past several years.
\end{abstract}

\section{MATERIALS AND METHODS}

Preliminary tests of compatibilities between workers of $R$. metallica taken from widely separated populations amply demonstrated:

Manuscript received by the editor November 8, 1979. 
(a) That there is indeed a sharp behavioral distinction between "home" and "alien" populations, and

(b) That $R$. metallica is sufficiently aggressive so that a simple confrontation of such "alien" workers, in the field, in the artificial nest, or simply in such "arenas" as laboratory finger bowls is sufficient to elicit sharp and unmistakable reactions at once. Thus, while observations involving compatibility reactions within and between colonies of some hypogeaic or semi-hypoeaic Ponerinae (such as members of the genus Amblyopone) must be undertaken with great care, and should be conducted within arenas, such as artificial nests, which are thoroughly familiar to the subjects and preferably have been occupied by them for many months beforehand, such precautions appear unnecessary with this active, aggressive epigeaic ectatomiine.

Three well-separated Australian populations of $R$. metallica were selected for the investigation. The first group was drawn from a rather small area in Southern Queensland, bounded by a rough triangle marked by the towns of Nambour, Montville, and Paramount Point, in the coastal Blackall Range. Nambour is approximately 59 miles north of Brisbane, and the distance between Nambour and Montville is approximately 7 miles. A second population was drawn from a limited area in Ashton Park, ${ }^{*}$ a municipal reserve on the outskirts of Sydney, N.S.W. separated by an arm of Sydney Harbor, and located approximately 560 miles from the Nambour-Montville region. This Ashton collection area included a roadside strip approximately one-half mile in greatest length by some sixty feet in width. The third area was located outside of the town of Sutherland, N.S.W., near the proximate border of the National Park of New South Wales, separated from the Ashton population by approximately 20 miles. It included strips of roadside verge approximately three miles in length by one-half mile in width.

Colonies were collected from these areas in 1959, 1963, 1968, 1973 , and 1977, and maintained in the laboratory in glass earthfilled Lubbock-type nests, kept in arenas where workers could forage at all times. Some individual colonies were maintained continuously under these conditions for as long as 14 years, so that, when desirable, reactions could be tested of workers more than one "generation" removed from the group originally collected.

*Now Sydney Harbor National Park 
Mode of Testing:

As mentioned, reactions of workers on meeting were usually immediate and quite clear cut, although some anomalies were observed. The procedure used was simply to introduce the individual to be tested into the colony tested against, or, alternatively, to place two workers together in a confined space, and to observe their behavior over periods ranging from less than a minute (when incompatibility was unquestionably evident, as in immediate and violent attack) up to an hour if necessary to be sure of compatibility. Reactions were arbitrarily (but, it is believed, reasonably accurately) scored in four "envelope" categories:

$A$. Extreme incompatibility, accompanied by attack, vigorous attempts to sting, and frequently tight body "closure".

$B$. Evident incompatibility, usually with seizure of antennae or legs, but without the violent "closure" of $A$. Both $A$ and $B$ were taken to mean marked incompatibility.

$C$. Initial "starting back" or alarm on first encounter, and sometimes brief running, but no threat or attack. The situation is commonly followed by fraternization and complete compatability.

$D$. Complete compatibility from the outset, lasting permanently, and frequently accompanied by vigorous antennal cleaning and sometimes mutual body licking. $C$ and $D$ may be justifiably interpreted, we believe, as ultimate compatibility, although obvious distinctions initially recognized in the $C$-type reactions were sometimes interesting.

Categories $A$ and $D$ were very clear cut. Inevitably, some observer subjectivity entered into assignments of $B$ and $C$ categories, but every effort was made to estimate them accurately. Very occasionally a reaction was sufficiently indefinite, or changed sufficiently with time, so that it had to be judged a "border case" and was so designated - as, for example " $C / D$ ".

The tests summarized below were designed to test several aspects of the whole question, as indicated.

\section{Controls}

Two sets of controls were run where the results to be expected were obvious, primarily to test the validity of the experimental conditions. One was between workers drawn from within a small contiguous area, the second between two fairly well-separated populations within a single collection location. 


\section{Ashton-Ashton}

Populations from three colonies (\#3, \#4, \#5), were collected at Ashton Park on January 3,1973, and tested on January 5. Colonies \#3 and \#4 were taken about 100 yards apart. Colony \#5 was separated from the other two by about $1 / 2$ mile. Following are the results: $\# 3 \times \# 4$ Test \#1-Divaricated mandibles, no attack. (C)

Test \#2-Same, close mutual examination, then mutual grooming, entire compatibility. (C)

$\# 3 \times \# 5$ Test \#1-Opened mandibles for a moment, then entire compatibility (D)

Test \#2-Opened mandibles, then entire compatibility. (D)

$\# 4 \times \# 5$ Test \#1-Entire compatibilty. (D)

Test \#2-Brief pursuit, with opened mandibles. (C)

\section{Sutherland-Sutherland}

Two colonies were used, taken in this case approximately 3 miles apart in the study area on January 23, 1973, and tested two days later, on January 5, 1973. They were labeled \#1 and \#2:

$\# 1 \times \# 2$ Test \#1-Entirely compatible (D)

Test \#2-Initial alarm with open mandibles and momentary seizure, passing to entire compatibilty (C)

\section{Ashton-Sutherland}

When workers from the two populations were tested against one another, the results were very different. These tests were performed on the same day as those above.

$\# 1 \times \# 3$ Test \#1-Extreme incompatibility, with active fighting

(A)

Test \#2-Extreme incompatibility. Pursuit but no seizure.

(B)

$\# 1 \times \# 4$ Test \#1-Continuous open-mandibled aversion (B)

Test \#2-Open-mandibled aversion. (B)

$\# 1 \times \# 5$ Test \#1-Open-mandibled aversion, no actual attack. (B) Test \#2-Continuous attacks, with frequent seizures (A)

$\# 2 \times \# 3$ Test \#1-Vigorous and continuing attacks (A)

Test \#2-Open-mandibled encounters frequently repeated (B) 
$\# 2 \times \# 4$ Test \#1-Instant attack and seizure (A) Test \#2-Open-mandibled encounters (B)

\#2 $\times \# 5$ Test \#1-Aggression; open-mandibled encounters (B) Test \#2-Immediate aggression and attack (A)

\section{SUMMARY}

Six tests among the Ashton populations were rated 3 each $\mathrm{C}$ and D. Two tests between Sutherland-location colonies were rated D and $\mathrm{C}$. There was thus a notable degree of compatibility among colonies drawn from within both study areas, although within the Sutherland area collections were made over a fairly wide range (about 3 miles apart).

In marked contrast, among 12 tests made between the Ashton and the Sutherland populations 5 were rated A, and 6 B. Incompatibility here was the general rule, varying only in the vigor of its expression.

Tests within a single "colony":

As a final test of the adequacy of the method, tests were run on the same day between workers taken in the same collection. The results were:

$\# 1 \times \# 1$ Entire compatibility (D)

$\# 2 \times \# 2$ Entire compatibility (D)

$\# 3 \times \# 3$ Entire compatibility (D)

$\# 4 \times \# 4$ Entire compatibility (D)

$\# 5 \times \# 5$ Entire compatibility (D)

It thus seemed clear (a) that the method itself was workable, and (b) that compatibility does diminish with distance, though "colonies" situated as much as three miles apart within the same study area (far beyond the likely bounds of daily worker encounters) can remain reasonably compatible, although at this distance there were often signs that workers were briefly "uncomfortable" in proximity, though they quickly adjusted. By contrast, members of widely separated populations (New South Wales and Queensland) uniformly behaved in an antagonistic fashion.

Is Compatibility a Conditioned or an Innate Perception and Reaction?

This question was approached in two ways:

(a) By introducing workers which had been eclosed from cocoons and had matured away from the parent "colony" (under 
the guardianship of one of two "home" adult workers) into that colony and noting the reactions of the "hosts".

(b) By testing reactions between colonies drawn from the same or different populations long enough after they had been separated (three to four years in most cases) so that at most a very small proportion of the workers living at the time of collection survived to participate. Thus the participants entirely or for the most part, had never been individually subjected to such tests. Thus, while the individual might itself have been conditioned by the distinctive colony odor of its own community, that distinction must persist over more than one worker generation. Results were as follows:

\section{Young Ants Returned to Parent Colony}

Young workers of $R$. metallica normally eclose from cocoons in a quite advanced stage of pigmentation and actively participate in colony functions within one or two days. This facilitated experimental procedures considerably, though the question remained open, of course, as to whether the "receiving" adults in the main community might distinguish their younger sisters or progeny on an age basis.

The colony used in this work (Y-1) had been collected from the Sutherland population on December 30,1959, and maintained as a laboratory group thereafter.

Test \#1

A group of cocoons from colony Y-1 was isolated on September 17, 1962, with 2 adult and 2 callow nurses.

On March 10, 1963, 20 workers (at least 18 of which must have been "strangers" to the home community) were reintroduced to the main colony with clean forceps. All were entirely compatible as shown.

Test \#1

$\begin{array}{cc}\text { Test No. } & \text { Reaction } \\ 1 & \text { Fully compatible (A) } \\ 2 & \text { Fully compatible (A) } \\ 3 & \text { Fully compatible (A) } \\ 4 & \text { Fully compatible (A) } \\ 5 & \text { Fully compatible (A) } \\ 6 & \text { Fully compatible (A) }\end{array}$




$\begin{aligned} 7 & \text { Fully compatible (A) } \\ 8 & \text { Fully compatible (A) } \\ 9 & \text { Fully compatible (A) } \\ 10 & \text { Fully compatible (A) } \\ 11 & \text { Fully compatible (A) } \\ 12 & \text { Fully compatible (A) } \\ 13 & \text { Fully compatible (A) } \\ 14 & \text { Fully compatible (A) } \\ 15 & \text { Fully compatible (A) } \\ 16 & \text { Fully compatible (A) } \\ 17 & \text { Fully compatible (A) } \\ 18 & \text { Fully compatible (A) } \\ 19 & \text { Fully compatible (A) } \\ 20 & \text { Fully compatible (A) }\end{aligned}$

Test \#2

On September 17, 1962, a second group of cocoons from Y-1 was isolated in the care of two adult nurses. They ecolosed and matured there.

On March 11, 1963, 17 workers were transferred with clean forceps, as before, back to the main colony. At the most, two of these workers (the two nurses) would previously have been exposed to the main group. In these tests, there was some low-order alarm as indicated, quickly passing to entire compatibility.

\section{Test \#2}

\section{Date Trial Reaction}

3/11 1 Slight hostility-opening of mandibles, some "starting back", no attack. Individual shortly accepted in home colony. (C)

$3 / 11 \quad 2 \quad$ Identical reaction to above. (C)

$3 / 113$ A little suspicion and obvious "starting" with open mandibles by two or three "home" individuals, but quick and complete acceptance. (C/D)

$3 / 1144$ Identical reaction to above (C/D)

$3 / 115$ Identical reaction to above. (C/D)

$3 / 126$ Identical reaction to above. (C/D)

$3 / 127$ Identical reaction to above. (C/D)

$3 / 12 \quad 8 \quad$ Identical reaction to above. (C/D)

$3 / 129$ Identical reaction to above. (C/D) 
3/12 10 Here the hostility was a bit more pronounced, many individuals "starting" with open mandibles, but no actual attacks occurring. (C).

$3 / 13 \quad 11 \quad$ Identical reaction to 9 . (C/D)

$3 / 14 \quad 12 \quad$ Identical reaction to 9 . (C/D)

$3 / 14 \quad 13 \quad$ Identical reaction to 9 . (C/D)

$3 / 14 \quad 14 \quad$ Identical reaction to 9 . (C/D)

$3 / 14 \quad 15 \quad$ Identical reaction to 9 . (C/D)

$3 / 14 \quad 16 \quad$ Identical reaction to 9 . (C/D)

$3 / 14 \quad 17 \quad$ Identical reaction to 9 . (C/D)

Experiments Repeated on October 7, 1962

10/7 18 Some "chasing," but no attack. Some "startle" effect. (C)

10/7 19 Identical to above. (C)

(b) Reactions Between Adult Members of the Same Populations After Several Years of Separation-Intervals Greater than the Average Life-span of Workers.

(b1) Ashton-Ashton

On January 4, 1973 a collection of two groups of workers was made from under adjacent stones at a specifically marked location in Ashton Park. They were separately colonized in the laboratory, and kept entirely isolated, being maintained under identical conditions of environment and diet on adjacent tables but not being permitted contact until March 20, 1977, more than four years later. These colonies were labeled $\mathrm{X}$ and 4 .

On March 2, 1977, an additional collection was made from precisely the same spot in Ashton Park, and brought to the laboratory. Tests among all three of these colonies were conducted on March 20, 1977, with the following results:

$\mathrm{D} \times \# 4$ Test \#1-Complete compatibility (D)

Test \#2-Complete compatibility (D)

$\mathrm{D} \times \mathrm{X} \quad$ Test $\# 1$-Complete compatibility (D)

Test \#2-Complete compatibility (D)

$4 \times \mathrm{X} \quad$ Test $\# 1$-Complete compatibility (D)

Test \#2-Slight initial "starting back", followed by complete compatibility. (C)

It is rather striking that after more than four years of separation, in one case between two populations in the laboratory maintained under identical regimens, in the other between these colonies and a 
wild population taken in the same location more than four years later, which must have existed under significantly divergent enviromental conditions, these three populations remained wholly compatible.

\section{(b2) Sutherland-Sutherland}

A similar series of four tests was made on January 17, 1973 between colonies collected in the same marked area within the Sutherland arena, respectively on January 12, 1968 (maintained thereafter in isolation) and January 3, 1973.

Test \#1-Initial "startle", followed by entire compatibility (C/D) Test \#2-Initial "startle", followed by entire compatibility (C/D) Test \#3-Initial "startle", followed by entire compatibility (C/D) Test \#4-Initial "startle", followed by entire compatibility (C/D)

(b3) "Blind" Tests between Individuals of Colonies from Sutherland and from Queensland in the Nambour-Montville-Paramount Point Triangle

These tests involved colonies collected in the Queensland area on December 23, 24, and 26, 1963, and a single colony collected in the Sutherland area on January 12, 1968. All had been housed since capture in earth-containing Lubbock nests in five-gallon aquaria with screened tops, as previously described, within a month of the time of collection. All had reared broods continuously over the nearly nine years that the Queensland collections were held before these tests, and the nearly five years for the Sutherland collection. During this time there had been numerous broods of males matured, and extensive male flights occurred within each arena, with presumed periodic re-fertilization of the successive broods of laying females. It is virtually certain that none of the workers tested from the Queensland collections were in existence when those collections were made, and extremely probable that the same was true for the Sutherland population.

For purpose of the tests, the locality label on each of the nests was obscured and replaced by an arbitrary designation, A1-A7 for the Queensland colonies, B1 for that from Sutherland.

A series of tests were run among the Queensland colonies and between them and the Sutherland colony, in all possible combinations, on October 23,1972. As a result, three compatibility groups could be distinguished. 
Queensland colonies A1; A2; A6; and A7 showed entire compatibility. They were designated as Group 1 .

Queensland colonies A3; A4; and A5 showed entire compatibility. They were designated as Group II.

Members of Group I and Group II were consistently incompatible inter se.

Members of both Group I and Group II were consistently incompatible with B1, the Sutherland population.

When the specific locality label for each colony was uncovered, it was found that the members of Group I (A1; A2; A6; A7) had been closely associated geographically when collected nine years before:

A1 and A2 had originally been taken a few feet apart at Paramount Point.

A6 had been taken a few feet from A1-A2 at Paramount Point.

A7 had been taken at Montville, a short distance away. Thus all of these had originally been parts of a closely circumscribed population.

The members of Group II (A3; A4; A5) had likewise been collected in close proximity, near the town of Nambour, approximately 7 miles from the first group.

These tests were repeated with the same colonies on March 20, 1977, when the Queensland colonies had been maintained in the artificial nest for more than thirteen years and that from Sutherland for more than nine years, and one of the Ashton colonies, collected January 4, 1973, was added. Colonies were renumbered as follows: $\# 1$ - Montville, Queensland (A7 in the October 23, 1972 tests recorded above.-Group I).

\#2- Nambour (Group II in 10/23/72 tests above).

$\# 3$ - Colony A1 in the 10/23/72 tests above (Group I).

\#4- " $\mathrm{X}$ " in the Ashton-Ashton tests described above. Ashton, January $4,1973$.

\#5- Sutherland, January 12, 1968 (B1 in the 10/23/72 test above).

$\# 1 \times \# 2$ (Old Group I $\times$ Group II)-Violent attack (A)

$\# 1 \times \# 3$ (Old Group I $\times$ Group I)-Complete compatibility (D)

$\# 1 \times \# 4$ (Old Group I $\times$ Ashton)

Test \#1-Violent attack (A)

Test \#2-Extreme avoidance (A/B)

$\# 1 \times \# 5$ (Old Group I $\times$ Sutherland)-Extreme "startle" reaction and avoidance (B) 
$\# 2 \times \# 3$ (Old Group II $\times$ Group I)-Violent attack (A)

$\# 2 \times \# 5$ (Old Group II $\times$ Ashton)-Agitation, mutual repulsion, no actual attack (B)

$\# 3 \times \# 4$ (Old Group I $\times$ Ashton)-Violent attack (A)

$\# 3 \times \# 5$ (Old Group I $\times$ Sutherland)-Violent attack (A)

$\# 4 \times \# 5$ (Ashton $\times$ Sutherland)

Test \#1-Violent attack (A)

Test \#2-Violent attack (A)

\section{SUMMARY AND CONCLUSIONS:}

These tests seem to point to three conclusions:

1. The capacity to make compatibility distinctions between wellseparated populations is highly developed in $R$. metallica, a normally aggressive species, despite the "diffuseness" of its colonial structure, characterized by the physical nature of the colonies themselves, the circumstance that communities consist only of monomorphic workers showing a minimum of morphological or habitus differentiation inter se even between ordinary and fertile laying workers, rather numerous in populous colonies.

However, consistent with this "looseness" of colony structure, groups of workers taken from the same general area but at distances clearly too great to permit continual communal contact under natural conditions retain some compatibility, the tolerance seeming to decrease with distance. Thus a picture of a rather "viscous" population seems more applicable in this context than that of well-defined colonial entities.

2. Compatibility distinctions were found to be consistently retained between groups originally drawn from the same natural populations but then isolated under identical laboratory conditions for up to more than thirteen years. Similar compatibility was exhibited between a group collected from a specific field location when tested shortly after capture against other groups collected at the same locality more than four years earlier and maintained in the laboratory over the interval under environmental conditions obviously very different. Thus differing environmental and nutritional histories do not seem to influence compatibility reactions in any observable way. 
3. Individuals eclosed apart from a given community and returned to it after maturity, though "strangers" to the parent community as individuals, are characteristically accepted as compatible. This suggests that the qualities affecting compatibility are here innately determined (cf. Crozier and Dix, 1979) though further work with "alien" nurses remains to be done.

All this suggests a genetic basis for the characteristics determining compatibility in $R$. metallica. The fact that the males of the species fly actively, and probably over some (though limited) distances, and mate, as we have demonstrated, with "calling" "laying workers" of neighboring colonies (Hölldobler and Haskins, 1977; Haskins, 1978), seems to fit this picture, suggesting an "interlaced" population, in which the frequencies of given matings between hypothetical colonies A and B diminish as some function of their spatial separation. The degree to which compatibility patterns can be maintained in isolated populations suggests that several generations (one would guess, of course, thousands or more) may be required to achieve significant shifts of compatibility.

\section{ADDENDUM*}

As a final test designed to distinguish genetic from environmental factors in colony-or population-discrimination in Rhytidoponera metallica, a colony of this species, collected in the Blackall Range at Montville, Queensland, Australia on December 23, 1963, and maintained in the laboratory since that time in a stacked group of modified, earth-containing Lubbock-type artificial nests in a plastic arena was divided into two approximately equal moieties by placing half of the nest stack in each of two identical plastic arenas arranged side by side on the laboratory bench. Conditions of temperature, light, and humidity were virtually identical for the two groups. Each remained in nests familiar to it, and each continued to breed normally during subsequent months. The only difference in treatment was in feeding. One moiety was given fresh mealworms at the rate of twice a week. The other was supplied with fresh crickets at the same intervals. The moiety given mealworms was supplied with dilute sugar water as a carbohydrate source; the "cricket fraction" with dilute honey.

*Addendum manuscript received by the editor September 22, 1980. 
The separation and differential feeding was begun on November 4, 1979, and continued until September 16, 1980. At that time individual pairs of workers from each moiety were tested for interactions in four inch fingerbowls, in the same manner as described in the main paper. Testing was carried out from 1:40 p.m. until 3:15 p.m. on September 16, 1980.

One hundred workers were tested, in fifty pairs of one from each moiety. Of these, 47 pairs, or 94 individuals, showed completely neutral (or "amicably interacting") reactions. In two pairs there was a slight initial hostile reaction, but no attack. In only a single case one individual seized the other by the base of an antenna, maintaining the grip for a few seconds before spontaneously releasing it with no further aggression.

As a control, workers from this same colony were tested on the same day against members of a colony of $R$. metallica collected at Sutherland, New South Wales, Australia, on January 12, 1968. These two colonies had been housed in similar Lubbock nests in arenas which had been maintained next to one another on the same laboratory bench for twelve years. Throughout this period, they had been fed identical diets: mealworms as a protein source, dilute sugar water as a source of carbohydrate. Thus diet, as well as all environmental conditions, had been maintained essentially identical for these two colonies over a twelve year period.

Seventeen pairs, involving thirty-four workers, were tested in the same manner as in the preceding case. In fifteen of these there was an almost instantaneous and violently hostile reaction, the pairs quickly becoming "locked" in a mutually stinging posture which quickly results in fatalities to one or both participants unless they are forcibly separated. In one case the members of a pair showed no reaction; in a second pair there was a distinct "startle" reaction when the two individuals met, but no actual attack.

The contrast between the behaviors of the members of a single genetically highly related population maintained on different diets in the one case and genetically separated populations maintained on identical diets in the other was very striking, and appeared to demonstrate quite unequivocally the predominant role of genetic factors in enabling colony (or "population") discrimination in this species, as against the role of nutritional factors, further reinforcing the conclusions drawn from the evidence presented in the main portion of the paper. 
The rare but conspicuous exceptions recorded in both series may hint at a multiple-allele genetic control mechanism, but further investigation and analysis of such a situation, if present, must await the future.

\section{Literature Cited}

Hölldobler, B. ANd C. P. HASKins

1977. Sexual calling in a primitive ant. Science 195: 793-794.

HASKINS, C. P.

1978. Sexual calling behavior in a highly primitive ant. Psyche: 85: 407-415. Crozier, R. H. AND M. W. Dix

1979. Analysis of two genetic models for the innate components of colony odor in social Hymenoptera. Behavioral Ecology and Sociobiology 4: 217-224. 

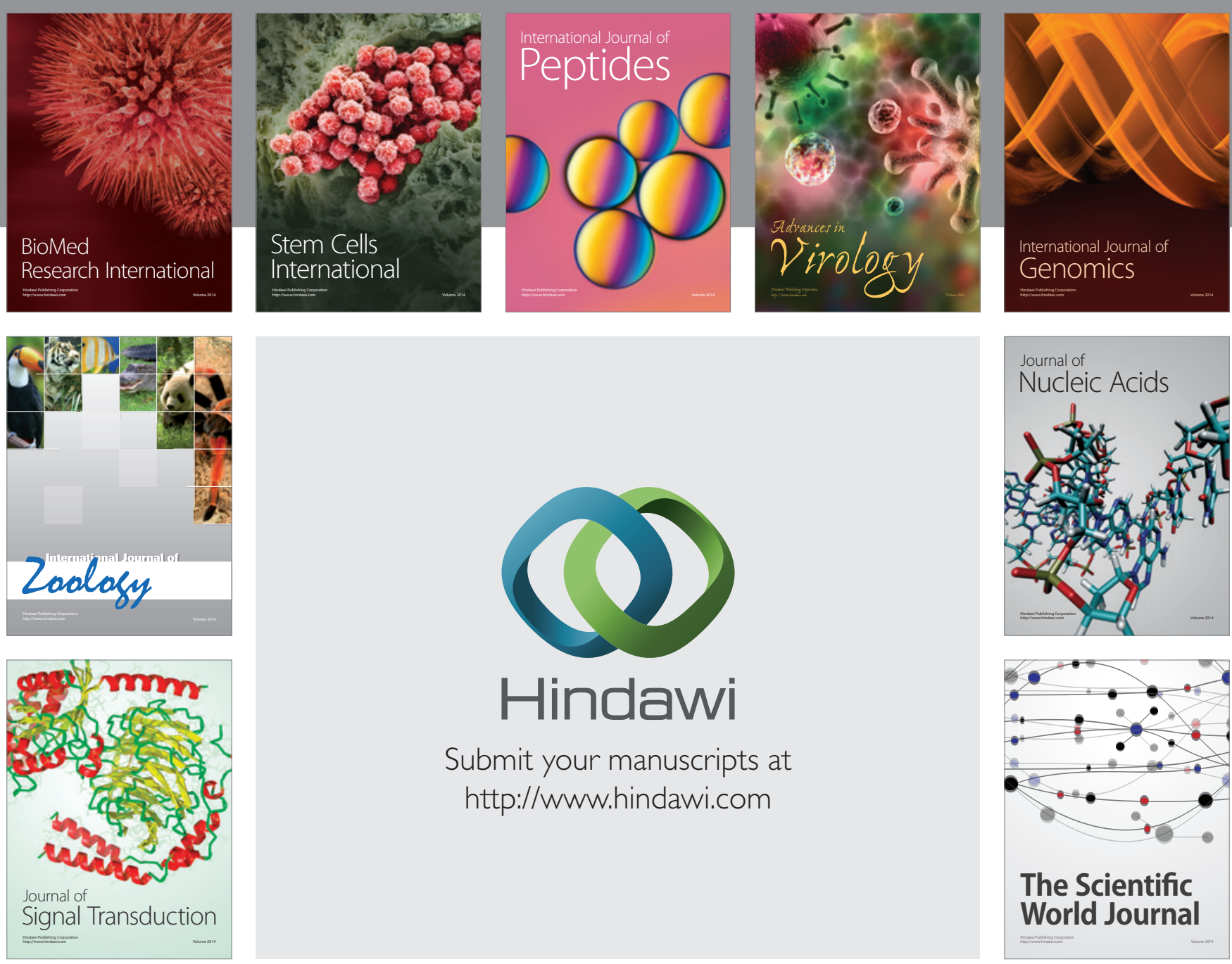

Submit your manuscripts at

http://www.hindawi.com
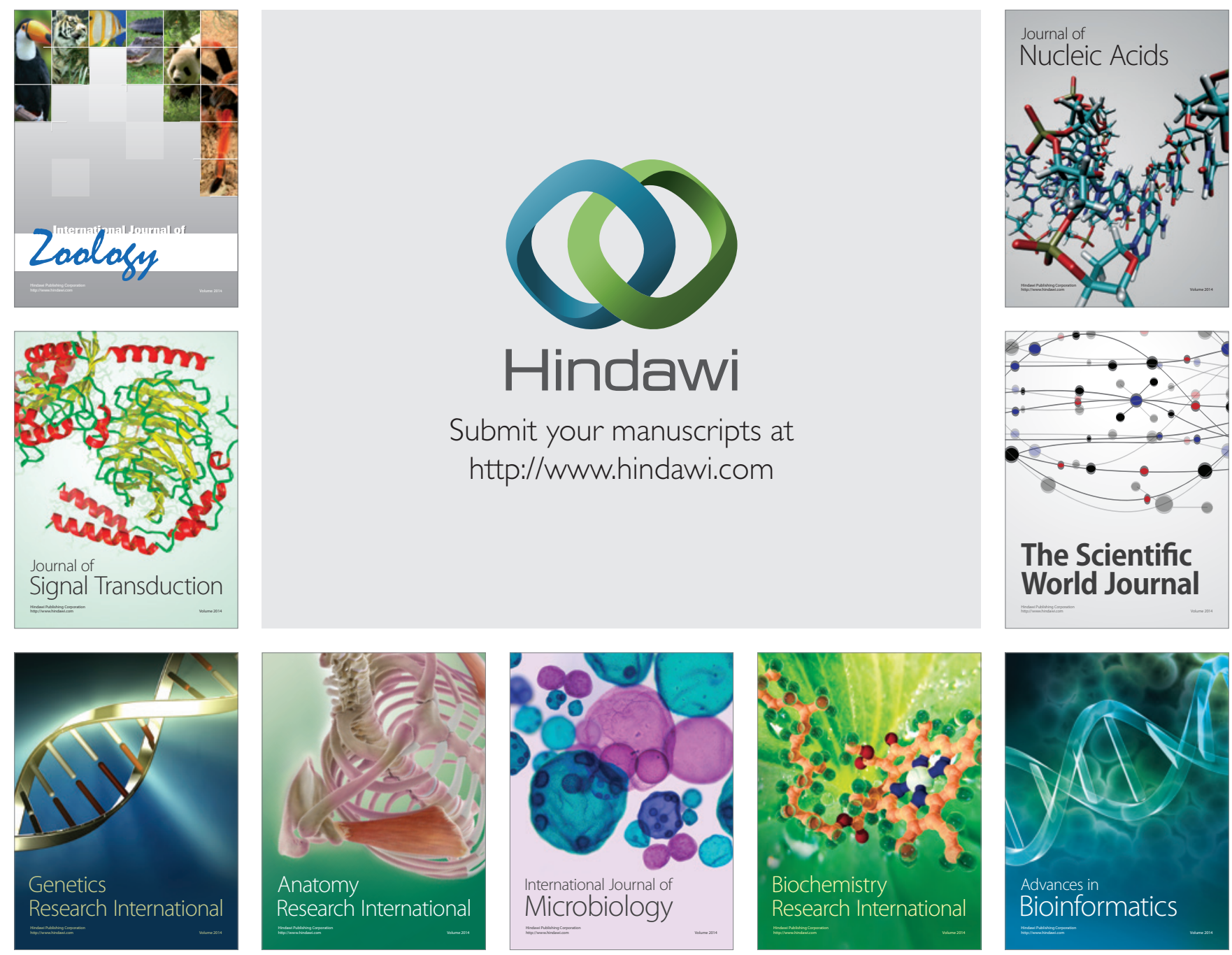

The Scientific World Journal
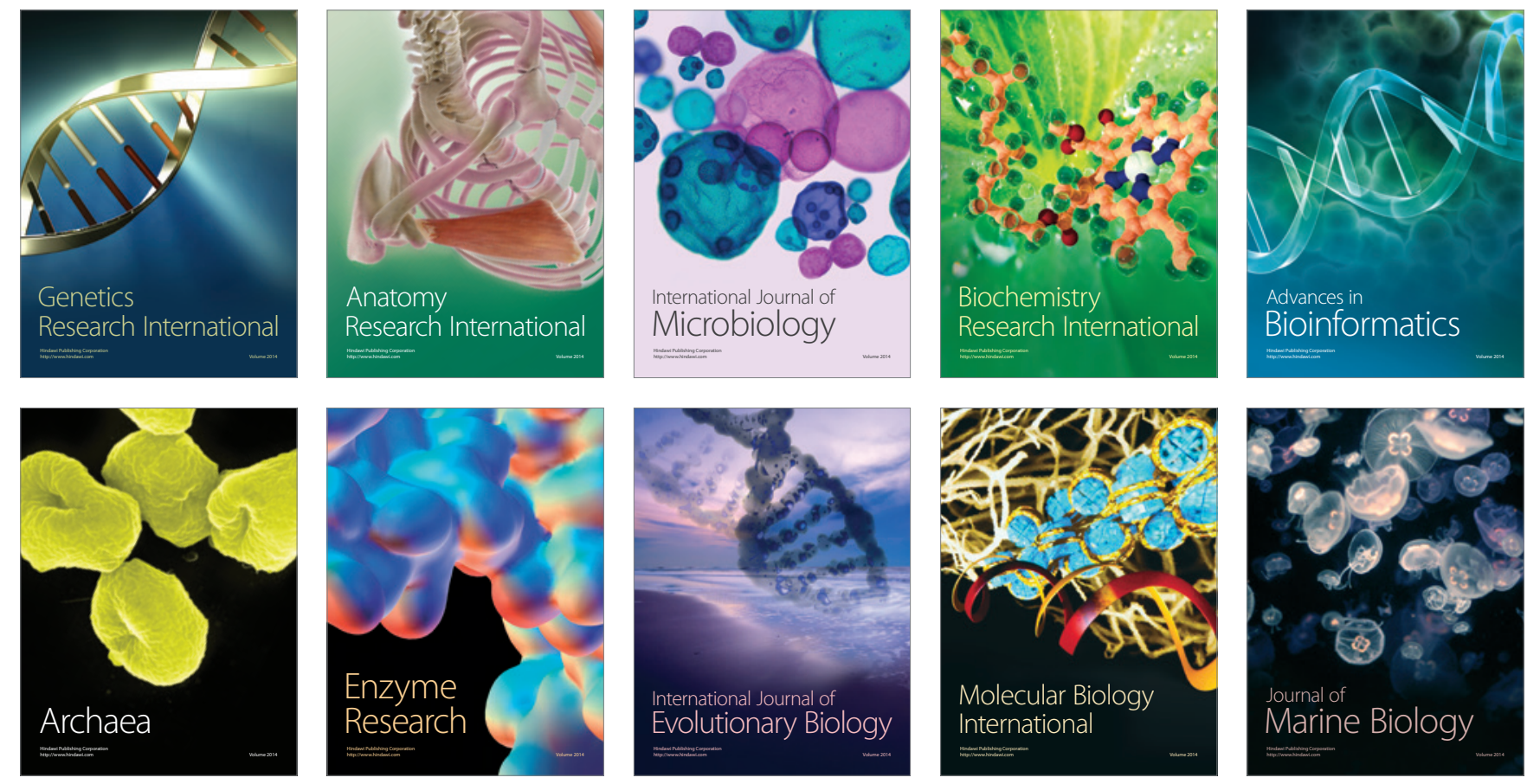